\title{
Model for Fluctuating Inflaton Coupling: Sneutrino Induced Adiabatic Perturbations and Nonthermal Leptogenesis
}

\author{
Anupam Mazumdar \\ CHEP, McGill University, Montréal, Quebec, Canada H3A 2T8
}

(Received 9 June 2003; published 15 June 2004)

\begin{abstract}
We discuss a unique possibility of generating adiabatic density perturbations and leptogenesis from the spatial fluctuations of the inflaton decay rate. The key assumption is that the initial isocurvature perturbations are created in the right-handed sneutrino sector during inflation which is then converted into adiabatic perturbations when the inflaton decays. We discuss distinct imprints on the cosmic microwave background radiation, which can distinguish nonthermal versus thermal leptogenesis.
\end{abstract}

DOI: 10.1103/PhysRevLett.92.241301

Inflation is the main contender for explaining the observed adiabatic density perturbations with a nearly scale invariant spectrum [1]. However, recently various alternative mechanisms for generating adiabatic density perturbations have been discussed, particularly converting the isocurvature perturbations of some light field into the adiabatic perturbations in the postinflationary Universe [2-4]. Another interesting proposal is that the perturbations could be generated from the fluctuations of the inflaton coupling to the standard model (SM) degrees of freedom $[5,6]$. It has been argued that the inflaton coupling strength to the ordinary matter, instead of being a constant, could depend on the vacuum expectation values (VEV) of various fields in the theory. These fields are none other than the flat directions of the minimal supersymmetric standard model (MSSM). However, the authors in [5] treated the flat directions without considering the fact that the flat directions are lifted at a nonrenormalizable level. In [7], the authors have demonstrated the importance of nonrenormalizable potential terms for the flat directions, which leads to dramatic changes in the estimation of the amplitude of the density perturbations in the original scheme. The amplitude of the perturbations dampens after the end of inflation, because the flat directions evolve after the end of inflation until the decay of the inflaton. The damping of the amplitude of the perturbations acts as a main challenge for realizing such a novel scheme (for a review on MSSM flat direction and cosmology, see [8]).

The idea is that if the MSSM condensates (made up of squarks and sleptons) are light during inflation then their quantum fluctuations can give rise to spatial fluctuations in the inflaton coupling strength. When the inflaton decays, the adiabatic density perturbations are created because the isocurvature perturbations generated by the flat direction during inflation are transferred to the adiabatic ones right at the time of decay. If the flat direction evaporates into baryons, it will give rise to the baryonisocurvature fluctuations, which can be constrained from cosmic microwave background radiation (CMB).

A particularly interesting implementation of this scenario is to consider the right-handed (s)neutrinos (super-
PACS numbers: $98.80 . \mathrm{Cq}, 12.60 . \mathrm{Jv}$

symmetry guarantees the presence of the right-handed sneutrino). If the right-handed (s)neutrino is Majorana, then it provides a natural explaining for the observed small neutrino masses via a seesaw mechanism, $m_{\nu} \approx$ $\left(m_{\mathrm{D}}^{2} / M_{N}\right)$ [9], where $m_{\mathrm{D}}$ is the Dirac mass obtained from the Higgs VEV.

The right-handed (s)neutrino is also a source for $L$ and/ or $B-L$ violation. Therefore it can be responsible for the observed baryon asymmetry. Leptogenesis requires $L$ or $B-L$ violating interactions, $C$ and $C P$ asymmetry, and an out of equilibrium condition. The first two are well served by the right-handed (s)neutrinos, and the last condition naturally arises in any inflationary cosmology.

Now let us briefly discuss the status of inflation. Undoubtedly, inflation is the most natural mechanism which makes the Universe homogeneous, flat, and isotropic. A single field slow roll inflation is also the most beautiful way of explaining adiabatic density perturbations. However, until now there has been hardly any connection between the inflationary sector and the known particle physics sector. In most of the cases, the inflaton is a gauge singlet, which leads to some degree of speculation on how the inflaton couples to the SM gauge particles, what the inflaton potential is, etc. The coupling of the inflaton to the SM fields is essential if the inflationary paradigm wishes to make connection to the hot big bang cosmology.

In this Letter, our aim is to present a simple toy model where we illustrate two important aspects. The first one is to consider the sneutrino induced isocurvature fluctuations which can generate adiabatic perturbations through inflaton decay. Second, we will show that in a nonthermal leptogenesis scenario the produced baryon-isocurvature fluctuations can be testable from CMB.

In this regard, it is natural to come up with a scenario where the inflaton couples only through the right-handed (s)neutrino sector. Note that the right-handed (s)neutrino acts as a mediator which connects the two disparate sectors, e.g., the inflaton and the SM via lepton (sleptons) and Higgs (Higgsinos). Therefore such a model is not only economical in terms of achieving density perturbations and lepton asymmetry, but also providing 
us with the correct relativistic species required for the nucleosynthesis.

For the purpose of illustration, let us consider a very simple toy model:

$$
W \supset \frac{1}{2} g \Phi \mathbf{N N}+h \mathbf{N H}_{u} \mathbf{L}+\frac{1}{2} M_{N} \mathbf{N} \mathbf{N},
$$

where $\Phi, \mathbf{N}, \mathbf{L}$, and $\mathbf{H}$, respectively, stand for the inflaton, the right-handed neutrino, the lepton doublet, and the Higgs (which gives mass to the top quark) superfields. Also, $M_{N}$ denotes right-handed (s)neutrino masses, and $g, h$ correspond to the Yukawas. Being a SM gauge singlet, the inflaton can naturally couple to the right-handed neutrino sector with a renormalizable and a nonrenormalizable coupling:

$$
g=g_{0}\left(1+\frac{\mathbf{N}}{M_{\mathrm{p}}}+\cdots\right),
$$

where we assume that the nonrenormalizable scale is the Planck mass, $M_{\mathrm{p}}=2.4 \times 10^{18} \mathrm{GeV}$. For simplicity, we have omitted all the indices in $h$ matrix and superfields, and we work on a basis where the Majorana mass matrix is diagonal. Further simplifications can be made for almost degenerate right-handed (s)neutrinos where $M_{N}$ is essentially the same for all. It is also conceivable in this case that the inflaton is coupled with the same strength to three right-handed (s)neutrinos with a mass hierarchy $M_{N} \gg m_{\phi}$, where $m_{\phi}$ can be treated as the mass of the inflaton in the minimum of its potential. Note that this is just a working example of nonthermal leptogenesis. We will highlight why we stress upon nonthermal leptogenesis compared to thermal leptogenesis. More complicated scenarios on nonthermal leptogenesis can be constructed $[10,11]$.

The inflaton sector is still unknown, except that it is responsible for driving inflation, which could be for, e.g., brane driven inflation, fast rolling inflation, kinetic driven inflation, assisted inflation, false vacuum inflation, etc. We further assume that the inflaton decays perturbatively. At this point, one might suspect that the above coupling, $g$, in Eq. (1), would give rise to a large mass contribution to the sneutrino through the inflaton VEV, and the sneutrino would simply roll down to the bottom of the potential. However, note that the inflaton VEV need not be always large in order to inflate; for instance, in a false vacuum inflation the inflaton could be fairly close to the bottom of its own potential. Nevertheless, let us imagine that we are working in a regime where the coupling, $g$, is such that the effective mass for the sneutrino is less than the Hubble expansion, $m_{\mathrm{eff}, \phi}<M_{\mathrm{eff}, N} \lesssim$ $H$, where $m_{\text {eff, } \phi}$ is the effective mass of inflaton during inflation. We will comment on the situation when the sneutrino masses are heavy compared to the Hubble expansion during inflation. From here onwards, we remove the subscript, eff.

An important point to note here is that during inflation the quantum fluctuations are created in the sneutrino sector, and the perturbations in the inflaton sector are assumed to be negligible; therefore, the perturbations arise only from the known particle physics sneutrino sector. The perturbations on a comoving scale larger than the Hubble scale can be foliated in terms of the curvature perturbations on a finite energy density surface: $d s^{2}=a^{2}(t)(1+2 \zeta) d x^{i} d x^{j}$, where $\zeta$ is a metric perturbation written in a proper coordinate system. In the presence of more than one scalar field, the total curvature perturbations $\zeta$ evolves outside the horizon due to nonvanishing pressure perturbations [12]:

$$
\dot{\zeta}=-\frac{H}{\rho+P} \delta P .
$$

where $\rho, P$ are the energy density and the pressure. For a single field inflation $\zeta=$ const, but in a multifield case $\delta P$ is a nonzero quantity due to the entropy perturbations, which can be defined in our case as

$$
S_{\phi, \tilde{N}}=3\left(\zeta_{\phi}-\zeta_{\tilde{N}}\right)=-3 H\left(\frac{\delta \rho_{\phi}}{\dot{\rho}_{\phi}}-\frac{\delta \rho_{\tilde{N}}}{\dot{\rho}_{\tilde{N}}}\right) .
$$

The overdot denotes differentiation with respect to coordinate time. Following our assumption, the initial entropy perturbation becomes $S_{\phi, \tilde{N}} \sim-3 \zeta_{\tilde{N}}$. For the Gaussian perturbations, we obtain

$$
\mathcal{P}_{\tilde{N}}^{1 / 2}=\frac{H_{*}}{2 \pi}
$$

where $*$ denotes when the interesting perturbations leave the horizon, $k=a_{*} H_{*}$. Note that the entropy perturbations feed the total curvature perturbations; therefore the entropy perturbations along with the individual perturbations, $\zeta_{\phi}, \zeta_{\tilde{N}}$, evolve in time. Though we do not prove this here, intuitively we can see that, in order to obtain the adiabatic density perturbations, the total curvature perturbation must become constant outside the horizon at the time of inflaton decay, when $\left.\zeta_{\phi}\right|_{\text {decay }} \sim \zeta_{\tilde{N}}$, and therefore $\zeta$ becomes constant on large scales, thus, converting its initial isocurvature fluctuations into the adiabatic ones.

Note that, besides the fluctuations in the sneutrino sector, there will be fluctuations in the Higgses and the sleptons also. However, their perturbations will not account for the baryon-isocurvature fluctuations in the above setup; for the time being we will neglect them.

The spectral index for the perturbations can be written as

$$
n_{\zeta}-1 \equiv \frac{d \ln \mathcal{P}_{\tilde{N}}}{d \ln k}=2 \frac{\dot{H}_{*}}{H_{*}^{2}}+\frac{2}{3} \frac{M_{N}^{2}}{H_{*}^{2}} .
$$

Therefore, as long as the Hubble expansion is slowly varying, and $M_{N} \leq H$, we can obtain a scale invariant density perturbation.

Now let us study the decay of the inflaton. The main decay mode of the inflaton is four-body final states consisting of two Higgs/Higgsino-lepton/slepton particles (and their CP transforms); this is due to the fact that the inflaton is decaying via off-shell (s)neutrino. The effective superpotential after integrating out $\mathbf{N}$ is given 
by

$$
W_{\text {eff }} \supset \frac{1}{2 M_{N}^{2}} g h^{2} \Phi\left(\mathbf{H}_{u} \mathbf{L}\right)\left(\mathbf{H}_{u} \mathbf{L}\right) .
$$

For simplicity, we may consider a situation when the (s)neutrinos are almost degenerate, e.g., $\Delta M_{N}<M_{N}$, and the Yukawa texture is such that the diagonal entries $(h)$ and off-diagonal entries $\left(h^{\prime}\right)$ follow $h^{\prime}<h$.

There are a total of nine final states: seven consist of two fermions and two scalars, and two consist of four scalars. Summing up all the final states, the decay rate and the final reheat temperature are given by

$$
\Gamma_{d} \simeq \frac{21 g^{2} h^{4} m_{\phi}^{5}}{2^{14} \pi^{5} M_{N}^{4}}, \quad \frac{T_{\mathrm{R}}}{m_{\phi}} \simeq \frac{10^{-7 / 2} g h^{2} m_{\phi}^{3 / 2} M_{\mathrm{P}}^{1 / 2}}{M_{N}^{2}} .
$$

However, note that the reheat temperature obtains spatial fluctuations due to Eq. (2):

$$
\frac{\delta T_{\mathrm{R}}}{T_{\mathrm{R}}}=-\frac{1}{3} \frac{\delta g}{g} \sim-\frac{\delta \tilde{N}}{3 M_{\mathrm{p}}} \sim-\frac{H_{*}}{6 \pi M_{\mathrm{p}}} .
$$

The factor $-1 / 3$ arises because during the decay of the inflaton the average energy density goes as $\rho \sim a^{-3}$ (for details, see [5,7]). For the Gaussian perturbations, $\tilde{N} \gg$ $H$, and following Eq. (5) we obtain the right amplitude for the density perturbations provided $H_{*} \sim 10^{-5} M_{\mathrm{p}}$. Note that there is no damping in the sneutrino fluctuations after the end of inflation in this case. Further, note that, if $\tilde{N} \ll H$, then the Gaussian amplitude of the perturbations will be damped [13], which we would like to avoid in this example.

Let us now obtain the lepton asymmetry in this model. The $C P$ asymmetry is obtained through the inference between the tree level and the one loop (vertex and selfenergy) corrected diagrams. Net $C P$ asymmetry in the off-shell case is quite different compared to the on-shell leptogenesis [10]. The self-energy correction comes out to be twice as much as the vertex correction for $m_{\phi} \ll M_{N}$. Final $C P$ asymmetry is then given by

$$
\epsilon_{C P} \simeq-\frac{3}{8 \pi} \times \frac{\sum_{i, n, l} \frac{\operatorname{Im}\left[\left(\mathbf{h h}^{\dagger}\right)_{n i}\left(\mathbf{h h}^{\dagger}\right)_{l l}\left(\mathbf{h h}^{\dagger}\right)_{i l}\right] m_{\phi}^{2}}{M_{i}^{3} M_{n}^{2} M_{l}}}{\sum_{i, n} \frac{\left(\left[\mathbf{h h}^{\dagger}\right]_{i n}\right)^{2}}{M_{i}^{2} M_{n}^{2}}},
$$

where $i, n, l=1,2,3$. The produced lepton asymmetry reads as

$$
\frac{n_{L}}{n_{\phi}} \simeq \frac{3}{\pi} \frac{\delta h h^{\prime 2}}{h} \frac{\Delta M_{N}}{M_{N}}\left(\frac{m_{\phi}}{M_{N}}\right)^{2},
$$

where $\delta h$ is nearly equal to diagonal entries of the Yukawa matrix. For the nearly degenerate case $\delta h / h \sim$ $\Delta M_{N} / 2 M_{N}$.

The total asymmetry in the baryons (after taking into account of the sphaleron effects) can be expressed as

$$
\eta_{\mathrm{B}}=\left(\frac{n_{B}}{n_{\phi}}\right)\left(\frac{n_{\phi}}{s}\right) \simeq \frac{1}{\pi} \frac{\delta h h^{2}}{h^{3}} \frac{\Delta M_{N}}{M_{N}}\left(\frac{M_{N} m_{\nu}}{\left\langle H_{u}^{0}\right\rangle^{2}}\right) \times\left(\frac{m_{\phi}}{M_{N}}\right)^{2}\left(\frac{T_{\mathrm{R}}}{m_{\phi}}\right),
$$

where $s=\left(2 \pi^{2} / 45\right) g_{*} T_{\mathrm{R}}^{3}$. Here $n_{\phi} / s$ denotes the dilution from reheating. By using the expression for the reheat temperature and $m_{\nu} \simeq\left(h^{2}\left\langle H_{u}^{0}\right\rangle^{2} / M_{N}\right)$, we finally obtain

$$
\eta_{\mathrm{B}} \simeq 4 \times 10^{-49 / 2} g \frac{\delta h h^{12}}{h^{3}} \frac{\Delta M_{N}}{M_{N}} \frac{m_{\phi}^{7 / 2} M_{\mathrm{P}}^{1 / 2}}{M_{N}^{2}\left\langle H_{u}^{0}\right\rangle^{4}}(1 \mathrm{GeV})^{2},
$$

where we have considered $m_{\nu} \approx 0.1 \mathrm{eV}$, and $\left\langle H_{u}^{0}\right\rangle=$ $174 \mathrm{GeV}$. If we demand degenerate light neutrino masses, then we can further simplify Eq. (13):

$$
\eta_{\mathrm{B}} \simeq \frac{2 \times 10^{-49 / 2} g h^{\prime 2}}{h^{2}}\left(\frac{\Delta M_{N}}{M_{N}}\right)^{2} \frac{m_{\phi}^{7 / 2} M_{\mathrm{P}}^{1 / 2}}{M_{N}^{2}\left\langle H_{u}^{0}\right\rangle^{4}}(1 \mathrm{GeV})^{2}
$$

For nearly degenerate heavy right-handed (s)neutrinos, with $M_{N}=10 m_{\phi}$ and $10^{-1} \leq h^{\prime} / h \leq 1$, we obtain the desired baryon asymmetry for $10^{-3} \leq g \leq 1$ and $10^{11} \mathrm{GeV} \leq m_{\phi} \leq 10^{13} \mathrm{GeV}$, which result in reheat temperature: $10^{6} \mathrm{GeV} \leq T_{\mathrm{R}} \leq 10^{8} \mathrm{GeV}$. At this point, one might worry upon the coupling strength, $g$, because the inflaton picks up an effective mass term, $g\langle\tilde{N}\rangle$, which has to be smaller than the inflaton mass, $m_{\phi}$, arising solely from the inflaton sector, in order to keep the successes of slow roll inflation. On the other hand, in order to generate the Gaussian fluctuations, $\tilde{N} \gg H_{*} \sim 10^{-5} M_{p}$ [see Eq. (9)]. This leads to $g \ll 1$; however, an exact magnitude of $g$ will depend on a particular inflationary model. Further, note that the reheat temperature is well below thermal and nonthermal gravitino overproduction $[14,15]$.

The most important point is to note that the baryon asymmetry is proportional to $g$ [see our final result, Eq. (14)]. Therefore baryons also feel the spatial fluctuations:

$$
\frac{\delta \eta_{B}}{\eta_{B}} \sim-\frac{1}{3} \frac{\delta g}{g} \sim-\frac{\delta \tilde{N}}{3 M_{\mathrm{p}}} \sim \frac{\delta T_{\mathrm{R}}}{T_{\mathrm{R}}} \neq 0 .
$$

The origin of $-1 / 3$ factor has the same origin as in Eq. (9). Note that the fluctuations in the baryon asymmetry are proportional to the fluctuations in the inflaton coupling, and therefore fluctuations in the reheat temperature. This shows that the baryonic asymmetry does not follow exactly the adiabatic density perturbations; instead perturbation in baryons is correlated baryon isocurvature in nature.

The baryon-isocurvature fluctuation leaves its imprint on cosmic microwave background radiation. Moreover, the fluctuations are correlated. The Wilkinson microwave anisotropy probe (WMAP) data provides a mild constraint on the correlated-cold dark matter (CDM)-isocurvature fluctuations [16], which can be translated in terms of the baryon-isocurvature fluctuations as $\left|S_{B} / \zeta\right|<0.32\left(\Omega_{\mathrm{CDM}} / \Omega_{B}\right) \sim 1.85$ at $95 \%$ confidence level, where $S_{B}$ is the baryon-isocurvature fluctuations. In our toy model $S_{B}=\delta \eta_{B} / \eta_{B}=$ $\delta T_{\mathrm{R}} / T_{\mathrm{R}}$. In particular, $\zeta=-H \delta \rho / \dot{\rho}=(1 / 4) \delta \rho_{\gamma} / \rho_{\gamma}=$ $\delta T_{\mathrm{R}} / T_{\mathrm{R}}$, where the subscript $\gamma$ denotes radiation. 
Therefore, we find $\left|S_{B} / \zeta\right|=1$ in our case, which is well within the WMAP constraint on the baryon-isocurvature perturbations.

We point out here that the above feature of correlated baryon-isocurvature perturbations is present only in a nonthermal case; this is the reason we pursued nonthermal leptogenesis. In nonthermal leptogenesis, there is an explicit dependence on the reheat temperature [see Eq. (12)]. This is indeed an interesting feature of a nonthermal leptogenesis which is absent in a thermal case. In a thermal leptogenesis the net asymmetry is proportional to a $C P$ asymmetry and not to a temperature [17]. We find that the constraints on the baryon-isocurvature perturbations can act as a tool for differentiating thermal versus nonthermal leptogenesis mechanisms.

Before we conclude our paper, we comment on a couple of interesting points. In an opposite limit, when $m_{\phi}>$ $M_{N}$, the inflaton decays via an on-shell right-handed (s)neutrino to the SM leptons and Higgs. This case is even better because during inflation the condition $\tilde{N} \gg$ $H_{*}$ is satisfied even better, because the sneutrino is lighter, $M_{N} \lesssim m_{\phi} \ll H$. However, one has to ensure that a thermal regeneration of the baryon asymmetry is really small. Finally, we comment on a heavy right-handed neutrino mass compared to the Hubble expansion. In this case, the sneutrino perturbations will be $\chi^{2}$ in nature, and usually the amplitude of the perturbations comes out to be small [13]. Nevertheless, they can also provide interesting imprints on CMB through the tilt in the spectral index. We leave this for future investigation.

In fact, we could also imagine perturbing the other Yukawa coupling, $h$, similar to Eq. (2), and the inflaton mass due to the fluctuations in the sneutrino VEV. By inspecting the reheat temperature, Eq. (8), and the baryon asymmetry, Eq. (12), we obtain $\delta T_{\mathrm{R}} / T_{\mathrm{R}}=$ $-(2 / 3)(\delta h / h)=\delta \eta_{\mathrm{B}} / \eta_{\mathrm{B}}$, assuming that the fluctuations are arising only from the diagonal elements of the Yukawas. In this case, the prediction on the baryonisocurvature fluctuations remains, $\left|S_{\mathrm{B}} / \zeta\right|=1$. However, the fluctuating inflaton mass gives rise to $\left|S_{\mathrm{B}} / \zeta\right|=1.4$, which is still within the WMAP limit on $\left|S_{\mathrm{B}} / \zeta\right|<1.85$ at 95\% confidence level [16].

As a final remark, there could be other sources for the isocurvature perturbations during inflation, including the most competitive candidate "cold dark matter." However, within supersymmetry excellent conditions arise naturally for their thermal production.

In summary, we point out that any supersymmetric leptogenesis scenario is a potential candidate for succeeding in generating the adiabatic density perturbations from the sneutrino fluctuations during inflation and generating the baryon asymmetry. The nature of perturbations (Gaussian/non-Gaussian) certainly depends on the mass scales, e.g., for $M_{N} \ll H_{*}$, and $\tilde{N} \gg H_{*}$ the perturbations are Gaussian. This may not be the case if $M_{N}=3 H$ during inflation (see [13]). Note that our model is eco- nomical because it achieves several goals at a time. Finally, we have found a very important benchmark which can potentially differentiate thermal versus nonthermal leptogenesis from CMB.

The author has benefited from the discussion with Rouzbeh Allahverdi, Robert Brandenberger, Zurab Berezhiani, Guy Moore, and David Wands.

[1] For a review, see A. D. Linde, Particle Physics And Inflationary Cosmology (Harwood, Chur, Switzerland, 1990).

[2] K. Enqvist, S. Kasuya, and A. Mazumdar, Phys. Rev. Lett. 90, 091302 (2003)

[3] K. Enqvist, A. Jokinen, S. Kasuya, and A. Mazumdar, Phys. Rev. D 68, 103507 (2003).

[4] K. Enqvist and M. S. Sloth, Nucl. Phys. B626, 395 (2002); D. H. Lyth and D. Wands, Phys. Lett. B 524, 5 (2002); T. Moroi and T. Takahashi, Phys. Lett. B 522, 215 (2001); 539, 303(E) (2002)]; S. Mollerach, Phys. Rev. D 42, 313 (1990); M. Postma, Phys. Rev. D 67, 063518 (2003).

[5] G. Dvali, A. Gruzinov, and M. Zaldarriaga, Phys. Rev. D 69, 023505 (2004).

[6] L. Kofman, astro-ph/0303614.

[7] K. Enqvist, A. Mazumdar, and M. Postma, Phys. Rev. D 67, 121303 (2003).

[8] For a review, see K. Enqvist and A. Mazumdar, Phys. Rep. 380, 99 (2003).

[9] M. Gell-Mann, P. Ramond, and R. Slansky, in Supergravity, edited by $\mathrm{P}$. van Niewenhuizen and D. Z. Freedman (North-Holland, Amsterdam, 1979); T. Yanagida, in Proceedings of the Workshop on the Baryon Number of the Universe and Unified Theories, Tsukuba, Japan, 1979, edited by O. Sawada and A. Sugamoto (KEK, Tsukuba, 1979); R. N. Mohapatra and G. Senjanović, Phys. Rev. Lett. 44, 912 (1980).

[10] R. Allahverdi and A. Mazumdar, Phys. Rev. D 67, 023509 (2003).

[11] Z. Berezhiani, A. Mazumdar, and A. Perez-Lorenzana, Phys. Lett. B 518, 282 (2001); R. Allahverdi, B. Dutta, and A. Mazumdar, Phys. Rev. D 67, 123515 (2003).

[12] V.F. Mukhanov, H. A. Feldman, and R. H. Brandenberger, Phys. Rep. 215, 203 (1992).

[13] A. D. Linde and V. Mukhanov, Phys. Rev. D 56, 535 (1997); A. R. Liddle and A. Mazumdar, Phys. Rev. D 61, 123507 (2000).

[14] J. Ellis, J. E. Kim, and D.V. Nanopoulos, Phys. Lett. B 145, 181 (1984)

[15] A. L. Maroto and A. Mazumdar, Phys. Rev. Lett. 84, 1655 (2000).

[16] H.V. Peiris et al., Astrophys. J. Suppl. Ser. 148, 213 (2003).

[17] In general, thermal/nonthermal leptogenesis provides the net asymmetry as $\eta_{B / L} \sim \epsilon_{C P} \times f\left(T_{d} / M\right)$, where $T_{D}$ is the temperature of the decaying particle, e.g., the righthanded neutrino. In the case of thermal leptogenesis $T_{d} \sim M$, and there is no temperature dependence. However, in a nonthermal case $T_{d} \neq M$. 